\title{
Las OTAs como elemento dinamizador de un destino turístico inteligente: procesos de integración y negociación con proveedores y clientes
}

\begin{abstract}
Grau Palomares, Sabrina
Escuela Politécnica Superior de Gandia, Universitat Politècnica de Valencia, Spain.

Teruel Serrano, M. a Dolores

Escuela Politecnica Superior de Gandia. Universitat Politècnica de Valencia, Spain.

(dteruel@upvnet.upv.es)

Palomares Chust, Alberto

Departamento de Sistemas Informáticos y Computación. Universitat Politècnica de Valencia, Spain.
\end{abstract}

\begin{abstract}
Resumen
En este trabajo se estudia el funcionamiento de las denominadas Agencias de Viajes Online (OTAs) que actualmente se han convertido en actores fundamentales para la dinamización de los Destinos Turísticos Inteligentes (DTIs). El propósito de este trabajo es conocer de qué manera, las OTAs logran llegar a sus clientes en un entorno altamente competitivo, y cómo utilizan las diferentes herramientas de marketing disponibles. El análisis detallado de un conjunto de OTAs (casos de estudio) ha permitido indagar en diferentes aspectos de su organización destacando, en particular, las características funcionales y organizativas que las diferencian de las agencias de viajes físicas tradicionales: la gestión interna y adquisición del producto, el funcionamiento del sitio web público, y las estrategias de marketing y comunicación con el cliente. Además este análisis ha permitido también identificar la relación entre las operaciones de negociación con proveedores y las condiciones de contratación que establecen en relación a la adquisición de los productos que ofrecen en sus sitios web.
\end{abstract}


Actas del Seminario Internacional Destinos Turísticos Inteligentes:

nuevos horizontes en la investigación y gestión del turismo

Universidad de Alicante, 26 y 27 de octubre de 2017

Palabras clave: agencias de viajes online (OTAs), comercio electrónico, distribución turística, integración XML, Destinos Turísticos Inteligentes (DTIS)

\section{Abstract}

In this work it is studied the operation of the called Online Travel Agencies (OTAs) which currently have become fundamental actors for the promotion of Smart Destinations. The purpose of this paper is to know in what way, the OTAs can reach out to customers in a highly competitive environment, and how to use the different marketing tools available. The detailed analysis of a set of OTAs (case studies) has made possible to investigate different aspects of its organization highlighting, in particular, the functional and organizational characteristics that differentiate them from the traditional physical travel agencies such as the internal management and acquisition of the product, the operation of the public web site, and the marketing strategies and communication with the customer..In addition, this analysis has also permitted to identify the relationship between the operations of negotiation with suppliers and the conditions of employment established in relation to the acquisition of the products OTAs offer on their web sites.

\section{Introducción y objetivos}

En los últimos años, la evolución de las Tecnologías de la Información y las Comunicaciones (TIC), ha propiciado un nuevo enfoque a la comercialización del sector turístico. Según las estadísticas de la comisión Nacional de los Mercados y la Competencia (CNMC, 2014), de las diez ramas de actividad con mayor porcentaje de volumen de negocio del comercio electrónico destacan las agencias de viajes y operadores turísticos y el transporte aéreo. Esta información nos da una idea de la importancia que tiene este tipo de empresas para el comercio electrónico.

Las Agencias de Viajes Online (en adelante OTAs ${ }^{1}$ ) son protagonistas esenciales de este cambio. El ámbito de actuación de las OTAs es el mercado global, y un sólo "click» las separa de sus competidores. Estas empresas presentan ventajas frente a las agencias de viajes físicas las cuales están condicionadas por su situación geográfica, y su competencia principal son otras agencias cercanas. Aunque la estructura y funcionamiento de las agencias de viajes tradicionales ha sido profundamente estudiada a lo largo de los

1. Siglas de su denominación en inglés: Online Travel Agency 
Actas del Seminario Internacional Destinos Turísticos Inteligentes:

nuevos horizontes en la investigación y gestión del turismo

Universidad de Alicante, 26 y 27 de octubre de 2017

últimos años y existe bastante literatura al respecto (Alcázar, 2002), apenas existen estudios o trabajos que aborden específicamente el fenómeno de las OTAs. Las OTAs son empresas turísticas, pero también son empresas tecnológicas, y la gestión de esa tecnología puede resultar tanto o más importante que la gestión del propio producto turístico. Así, este nuevo modelo de negocio presenta unas características propias, tanto en su funcionamiento interno, como en el proceso de adquisición de productos y servicios turísticos, o en relación a las estrategias de marketing y comunicación con los clientes.

En relación a los destinos turísticos inteligentes (DTI), las agencias de viajes (tanto online como offline) constituyen un elemento importante en la medida en que, además de su papel intermediador y comercializador de la oferta turística, actúan como inspiradoras y persuasoras del turista previo a la organización del viaje. Es cierto que las OTAs no venden un destino en exclusiva (a no ser que se dediquen a la actividad receptiva) pero a pesar de esto, la contribución que hacen a los destinos es muy alta en términos de reservas y de flujo de turistas. En este sentido, y a modo de ejemplo, simplemente realizando una búsqueda sencilla de "un hotel en Benidorm» Google ofrece varios millones de resultados, y entre los primeros, encontramos que la mayoría son OTAs. Este dato nos indica que estas empresas se mueven en un mercado altamente competitivo por lo que la diferenciación en producto, atención al cliente, accesibilidad del sitio web o el precio final son vitales para su éxito.

Por último, cabe destacar que la actividad turística en general y los negocios turísticos vinculados a los viajes presentan una alta dependencia a la información que debe estar actualizada, bien presentada y precisa. Así, en el entorno de un DTI, las OTAs contribuyen a los destinos por tratarse de un elemento fundamental para su promoción y comercialización y para ello debe propiciar el establecimiento de relaciones entre el destino y estas empresas incluso facilitarles visibilidad a través de enlaces en el sitio web del destino. Por esta razones, el objetivo que se plantea es definir y analizar las particularidades organizativas y de funcionamiento de las agencias de viajes online para clarificar los procesos que se establecen y a partir de ellos analizar los actores implicados. Asimismo, se ahondará en el conocimiento de la contribución de estas empresas en la dinamización turística de un destino inteligente en base a los indicadores y criterios de diagnóstico de aplicación a los DTI (GVA, 2016). 
Actas del Seminario Internacional Destinos Turísticos Inteligentes:

nuevos horizontes en la investigación y gestión del turismo

Universidad de Alicante, 26 y 27 de octubre de 2017

\section{Metodología}

La metodología utilizada para la realización de esta investigación se fundamental en el análisis cualitativo de una muestra representativa de las OTAs a nivel del territorio español. Se han elegido 10 ejemplos (casos de estudio), considerando como criterios de selección su volumen de negocio y el número de empleados. La información se ha obtenido de la base de datos oficiales del sistema de análisis de balances $\mathrm{SABI}{ }^{2}$.

De acuerdo con estos criterios, los casos de estudio analizados se recogen en la Tabla 1:

\begin{tabular}{|l|l|}
\hline \multicolumn{1}{|c|}{ OTAs pequeñas } & \multicolumn{1}{c|}{ OTAs grandes } \\
\hline $\begin{array}{l}\text { Facturación }<5.000 .000 € \\
\text { Empleados }<50\end{array}$ & $\begin{array}{l}\text { Facturación }>5.000 .000 € \\
\text { Empleados }>50\end{array}$ \\
\hline Roomsday.com & Centraldereservas.com \\
\hline Bookaris.com & Logitravel.com \\
\hline Aviajes.com & Atrapalo.com \\
\hline Quierohotel.com & Muchoviaje.com \\
\hline Quehoteles.com & Destinia.com \\
\hline
\end{tabular}

Tabla 1: Relación de OTAs estudiadas en función del tamaño y facturación

Esta investigación se ha realizado con la elaboración de una encuesta a través de un cuestionario que ha servido para determinar algunos aspectos referidos a la organización interna y a las estrategias de marketing y comunicación de las 10 OTAs seleccionadas con el cliente.

Además se mantuvieron entrevistas en profundidad con 2 directivos que permitieron corroborar los datos obtenidos a través de las encuestas. Adicionalmente se consultaron los sitios web de los casos de estudio, con el fin de recopilar datos del proceso de adquisición de los productos ofertados y sobre el funcionamiento del sitio web orientado al cliente.

2. Se han utilizado datos del año 2013 que eran los que estaban disponibles en la Base de Datos SABI al realizar este estudio. 
Actas del Seminario Internacional Destinos Turísticos Inteligentes:

nuevos horizontes en la investigación y gestión del turismo

Universidad de Alicante, 26 y 27 de octubre de 2017

En relación a la contribución de las OTAs a los DTI, este trabajo revisa el autodiagnóstico/estudio reciente presentado por la GVA, según el cual las OTAs se incluirían en el ámbito dedicado a la Innovación y concretamente en el indicador "Penetración de TICs en empresas turísticas». Este indicador evalúa el uso de las TICs por parte de las empresas turísticas del destino en acciones de marketing online, social media y comercialización y se le concede una ponderación del $15 \%$.

\section{Caracterización de OTAs}

Las OTAs se ven afectadas también por la legislación referente al e-commerce, ya que su actividad se desarrolla en este medio. En concreto, la ley 34/2002 de Servicios de la Sociedad de la Información y de Comercio Electrónico regula los aspectos básicos de e-commerce, contratación on-line e información y publicidad entre otros. Los artículos que cabe considerar como más importantes de esta ley se refieren a aspectos formales de información obligatoria que la empresa explotadora de un sitio web con fines comerciales debe tener como son: nombre de la empresa, domicilio, datos de inscripción en el registro mercantil o datos de contacto (art. 10).

Por otro lado, la prohibición del «SPAM» o correo no deseado salvo que hubiera sido solicitado de manera expresa por el consumidor (art. 21) o la regulación del uso de cookies exigiendo el consentimiento del usuario informando previamente del uso de dichas cookies (art. 22).

Asimismo, como empresas turísticas de intermediación su regulación se haya recogida en el título IV del Texto Refundido de la Ley General para la Defensa de los Consumidores y Usuarios y Otras Leyes Complementarias donde se define la naturaleza de estas empresas indicando que tienen la consideración de agencias de viajes las personas físicas o jurídicas que, sin perjuicio de la realización de cualquier actividad de mediación y/u organización de servicios turísticos, se dedican a la organización y venta de los denominados viajes combinados. Las agencias de viajes tanto físicas como online, son empresas dedicadas a la intermediación y venta de productos y servicios turísticos. Obviamente para poder ofrecer y vender estos servicios al cliente final deben disponer de la información sobre las características de los productos, disponibilidad, precios así como la posibilidad de hacer la reserva de los mismos.

En una agencia de viajes física, la función asesora es crucial para poder realizar la venta posterior, por ello los catálogos publicados por los 
Actas del Seminario Internacional Destinos Turísticos Inteligentes:

nuevos horizontes en la investigación y gestión del turismo

Universidad de Alicante, 26 y 27 de octubre de 2017

proveedores (turoperadores, compañías de cruceros, etc.) donde consultar la información y precios de cada servicio son fundamentales. La confirmación y reserva se realiza a través del sitio web del proveedor o bien por teléfono con el departamento de reservas del mismo. Asimismo, en el caso de compañías aéreas se utilizan GDS o consolidadores aéreos ${ }^{3}$.

En el caso de las OTAs, el proceso de información y venta no se realiza igual, debido a que el cliente realiza la búsqueda de información, precios y disponibilidad de manera autónoma. No obstante, esta falta de asesoramiento y dada su importancia en el proceso de venta, hace que algunas OTAs ofrezcan un servicio de chat para dar soporte al proceso de venta. Se trata por tanto de un proceso complejo ya que la asesoría y selección de productos que hace la OTA dependen de la pericia de un agente de viajes quien previamente ha contratado diferentes productos y servicios. Para entender mejor el proceso de adquisición del producto por parte de las OTAs analizaremos: i) la contratación del producto, ii) la política de precios y cláusulas de paridad, y iii) los canales de adquisición del producto.

\subsection{Proceso adquisición de producto y/o servicio}

En general, la contratación del producto turístico se realiza, o bien directamente con el proveedor del servicio (hoteles, empresas de alquiler de coches, compañías aéreas, etc.) o bien a través de intermediarios (turoperadores, consolidadores aéreos, empresas de receptivo, etc.). En los últimos años, las OTAs están alterando los equilibrios de poder en la cadena de distribución turística debido a su alto volumen de ventas, restándole protagonismo incluso a los turoperadores. Los volúmenes de plazas que manejaban estas empresas tenían un tamaño desproporcionado respecto a cualquier agencia de viajes por lo que se veían obligadas a integrarse a través de franquicias, grupos de gestión o grandes redes con el fin de mejorar su posición competitiva. Sin embargo, actualmente las OTAs de tamaño grande/medio igualan o superan en volumen de negocio a los turoperadores, por lo que se están convirtiendo en el actor más relevante y con mayor capacidad de negociación en el sector, consiguiendo tarifas y condiciones muy ventajosas (rappels, plazos de pago, etc.) (Canalis, 2013).

3. Un consolidador aéreo es una agencia de viajes virtual que aglutina y comercializa la oferta de varias compañías aéreas. Al manejar grandes volúmenes de plazas de avión consiguen mejores precios. 
Actas del Seminario Internacional Destinos Turísticos Inteligentes:

nuevos horizontes en la investigación y gestión del turismo

Universidad de Alicante, 26 y 27 de octubre de 2017

\subsection{Política de precios y cláusulas de paridad}

El segundo aspecto del proceso de adquisición de productos por parte de las OTAs es el que se refiere a la política de precios y cláusulas de paridad. Las agencias de viajes físicas trabajan con un modelo de precio venta al público (PVP) comisionable donde, el proveedor del producto y/o servicio marca el precio de venta y proporciona una comisión a éstas por la venta. Sin embargo las OTAs trabajan con precios netos a los que se le añade un margen comercial (denominado Markup). El alto volumen de reservas que gestionan de las OTAs y la competitividad existente entre ellas hace que el Markup se vea reducido, ya que la mayoría de las OTAs ofrecen un precio inferior al del proveedor "canibalizando» ${ }^{4}$ las ventas directas del propio proveedor.

Ante esta práctica, extendida a nivel internacional, los proveedores imponen políticas de paridad de precios al objeto de respetar el PVP ofrecido por el proveedor y visible en su sitio web. En el caso de que la OTA incumpla dicha paridad, el proveedor puede cancelar la relación comercial.

Para analizar si las OTAs respectan la paridad de precios, hemos comparado los resultados de las búsquedas realizadas en los sitios web de las OTAs (10 casos de estudio) para un mismo producto (Hotel 1 y Hotel 2$)^{5}$ y en las mismas fechas, con el precio ofrecido desde el propio sitio web de dichos hoteles. Los resultados se muestran en la tabla 2:

\begin{tabular}{|l|c|c|c|c|c|c|c|c|c|c|c|}
\hline Agencias & Web & $\mathbf{1}$ & $\mathbf{2}$ & $\mathbf{3}$ & $\mathbf{4}$ & $\mathbf{5}$ & $\mathbf{6}$ & $\mathbf{7}$ & $\mathbf{8}$ & $\mathbf{9}$ & $\mathbf{1 0}$ \\
\hline Hotel $\mathbf{1}$ & $1.638 €$ & $1.639 €$ & $1.638 €$ & $1.638 €$ & $1.661 €$ & $1.639 €$ & $1.638 €$ & $1.638 €$ & $1.638 €$ & $1.638 €$ & $1.638 €$ \\
\hline Hotel $\mathbf{2}$ & $983 €$ & $865 €$ & $861 €$ & $904 €$ & $870 €$ & $891 €$ & $853 €$ & $871 €$ & $832 €$ & $833 €$ & $853 €$ \\
\hline
\end{tabular}

Tabla 2: Comparativa precios. Web propia y OTAS

Estos resultados obtenidos en la búsqueda del hotel 1 confirman que todas las agencias están respetando el mismo precio que oferta el propio hotel en su web. Se puede deducir que este hotel utiliza políticas de paridad de precios. Sin embargo en la búsqueda del hotel 2 , se observa que todas las OTAs analizadas ofrecen un precio inferior al del propio hotel y a su vez ofrecen un precio diferente entre ellas. En este caso las OTAs ofrecen un

4. En el argot de las OTAs a esta práctica se le ha denominado «canibalizar los precios».

5. Por razones de confidencialidad no se indican los nombres de los 2 hoteles analizados. 
Actas del Seminario Internacional Destinos Turísticos Inteligentes:

nuevos horizontes en la investigación y gestión del turismo

Universidad de Alicante, 26 y 27 de octubre de 2017

descuento medio del $12 \%$ sobre el PVP del hotel, beneficiando así el propio consumidor pero perjudicando al proveedor del servicio, que deja de controlar sus propios precios.

\subsection{Integraciones tecnológicas}

En este apartado se describen las diferentes formas utilizadas por las OTAs para la integración de los diferentes productos turísticos en sus páginas web para su posterior venta al cliente. Estas integraciones tienen su origen en los denominados Sistemas Globales de Reservas (GDS en inglés) para la venta principalmente de plazas aéreas y hoteles. También son utilizados para la venta de otros productos turísticos como puede ser la reserva de coches de alquiler, aunque en menor medida. Actualmente se siguen usando principalmente para el producto aéreo.

En la modalidad denominada carga manual, la información sobre disponibilidad y precios se encuentra alojada directamente en los servidores de la OTA. Suele estar asociada a la contratación directa entre hotel y la OTA aunque no exclusivamente. Esta información puede ser cargada de forma manual por el personal del departamento de producto de la OTA, si bien esto genera mucha carga de trabajo para la agencia, que además debe ir actualizando la información constantemente.

No obstante, para reducir esta carga de trabajo, las OTAs utilizan herramientas denominadas extranets, a través de las cuales el proveedor de servicios puede gestionar sus operaciones, y través de su cuenta puede añadir imágenes, descripción de sus productos, actualizar sus tarifas y disponibilidad e incluso añadir ofertas especiales. Así, aunque sigue siendo un proceso laborioso, no lo es tanto para la OTA que reparte el trabajo entre todos sus proveedores de servicios. Este canal es minoritario, pues según los datos obtenidos del cuestionario realizado a las OTAs, se ha podido determinar que sólo un $11,42 \%$ de las ventas de las agencias estudiadas provienen de este sistema.

La modalidad denominada integración XML funciona de conector a modo de "traductor» entre clientes y proveedores, permitiendo que distintos sistemas informáticos puedan ser compatibles entre sí. Una integración XML es una conexión informática entre un cliente y un proveedor que se realiza utilizando el lenguaje de marcas XML. Esta conexión conecta los sistemas informáticos permitiendo el envío y recepción de información en tiempo real. Este nuevo canal supone una gran diferencia respecto a las formas 
Actas del Seminario Internacional Destinos Turísticos Inteligentes:

nuevos horizontes en la investigación y gestión del turismo

Universidad de Alicante, 26 y 27 de octubre de 2017

anteriores de distribución turística dado que todo el proceso (petición de precios y disponibilidad, reservas y cancelaciones) se realiza en tiempo real y sin la necesidad de la intervención de ningún operador o agente de viajes. De esta manera es posible disponer de la información de un proveedor de hotel, un Rent a Car, una aerolínea, una compañía ferroviaria o una naviera desde el sitio web de las OTAs, aunque sus sistemas informáticos sean muy diferentes.

Existen dos tipos de integraciones XML:

- Integraciones PULL: En esta modalidad la OTA no tiene los precios y disponibilidad de los servicios en sus servidores sino que cuando un cliente realiza una consulta en la web, conecta en tiempo real con los diferentes proveedores para consultar esta información. Es decir cada vez que un cliente hace una búsqueda en una OTA, ésta «pregunta» a los proveedores por la disponibilidad y precios.

- Integraciones PUSH: En esta modalidad es el proveedor el que proporciona la disponibilidad, precios y resto de información a la OTA previamente a las consultas de los clientes. El proveedor se tiene que encargar de ir actualizando la información de la que dispone la OTA. Es un sistema parecido a la carga manual (la información reside en la OTA) pero se realiza de forma automatizada a través de conexiones $\mathrm{XML}$ y no de forma manual. Cuando un cliente hace una búsqueda en la OTA, ésta consulta la información que tiene en su propio servidor y que ha sido actualizado previamente por el proveedor.

A modo de ejemplo, en la figura 1 se muestra un pequeño extracto de una trama XML (dado que la trama completa es muy extensa) facilitada por una de las OTAs estudiadas. En este caso aparece la respuesta del turoperador a la solicitud de la OTA, en la que se ofrece disponibilidad y tarifas de diferentes establecimientos en el destino solicitado: 
Actas del Seminario Internacional Destinos Turísticos Inteligentes:

nuevos horizontes en la investigación y gestión del turismo

Universidad de Alicante, 26 y 27 de octubre de 2017

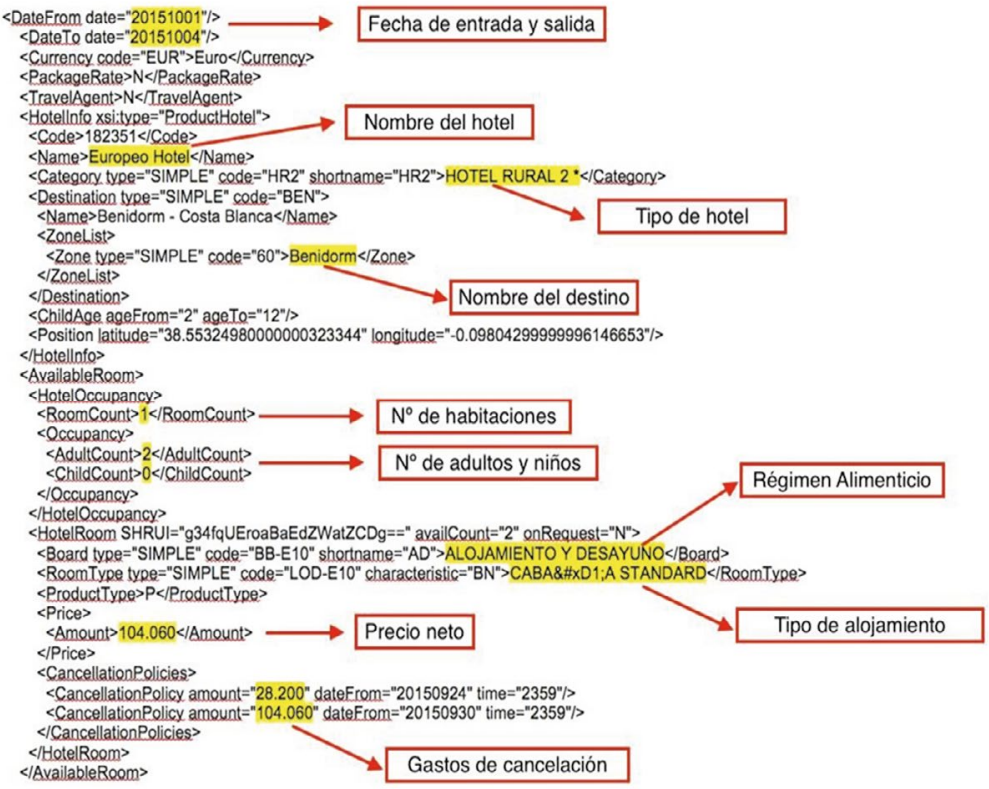

Figura 1: extracto de una trama XML

Actualmente las integraciones XML son la tecnología preferentemente utilizada por las OTAs para la adquisición de producto. De acuerdo con los datos obtenidos, el $89 \%$ de las OTAs obtienen los productos a través de estas integraciones.

Sin embargo, aunque las ventajas de las integraciones $\mathrm{XML}$ son obvias, también tienen inconvenientes. El principal problema detectado es la dispersión de los estándares ya que cada empresa utiliza su propio desarrollo. Esto provoca que cuando dos empresas quieren conectar sus sistemas deben dedicar unos recursos considerables a hacer un desarrollo específico.

Para intentar solucionar este problema surgen iniciativas como la Open Travel Alliance, que es una organización fundada en 1999 en la que participan empresas turísticas de todos los sectores y empresas tecnológicas. Desde esta organización se desarrollan especificaciones y estándares XML abiertos que permiten la rápida interconexión de las empresas que utilicen dichos estándares. Este objetivo se logra a medias porque no todos los 
Actas del Seminario Internacional Destinos Turísticos Inteligentes:

nuevos horizontes en la investigación y gestión del turismo

Universidad de Alicante, 26 y 27 de octubre de 2017

agentes turísticos los siguen, y también porque el propio estándar recoge muchas particularidades, lo que hace que merme su eficacia tal y como recoge Huerta (2016).

Otra opción más extendida y en algunos casos patrocinada por las administraciones públicas son los denominados Channel Manager. Los Channel Managers distribuyen el producto hotelero a turoperadores, OTAs y SGDs. Así el hotel actualiza la información sobre cupos, tarifas, disponibilidad, en un único panel de su Channel Manager, y es éste el que actualiza toda la información (mediante conexiones XML Push o Pull) a los diferentes proveedores.

Además, el Channel Manager se puede conectar con su sistema de gestión de hoteles también denominado Property Management System (más conocido por sus siglas del inglés - PMS). Esta conexión se puede realizar de manera unidireccional o bidireccional. En el primer caso el hotel sólo tiene que mantener su inventario en su propio programa de reservas y éste se conecta con el Channel Manager que a su vez se conecta a los proveedores. La conexión es bidireccional cuando un proveedor realiza una reserva, ésta llega al Channel Manager y de ahí se integra directamente en el PMS. Es decir la reserva de un cliente queda directamente registrada en el programa del hotel sin intervención de un operador.

La integración tecnológica de las empresas a través de los Channel manager es tan importante para la distribución turística que diversas iniciativas han sido impulsadas por las administraciones públicas. Un caso especialmente notorio es Travel Open Apps, creado por la Generalitat Valenciana en el año 2011. Esta plataforma es un Channel Manager gratuito para todas las empresas turísticas de la Comunidad Valenciana. Según el artículo publicado en Hosteltur (Hosteltur, 2014) Travel Open Apps gestionó durante el año 2014 más de 43.000 reservas con una facturación de 23,5 millones de euros.

La Comisión Europea se ha basado en la experiencia y la tecnología de Travel Open Apps para su proyecto Tourismlink (European Commission, 2012) que consiste en la creación de un Channel Manager para interconectar todas las empresas turísticas de la Unión Europea, y que aspira a ser un auténtico estándar a nivel europeo.

Todo el proceso de adquisición del producto que se ha descrito en este apartado mediante cargas manuales, a través de extranets, integraciones XML y Channel Manager se representa en el siguiente esquema (Fig. 2): 
Actas del Seminario Internacional Destinos Turísticos Inteligentes:

nuevos horizontes en la investigación y gestión del turismo

Universidad de Alicante, 26 y 27 de octubre de 2017

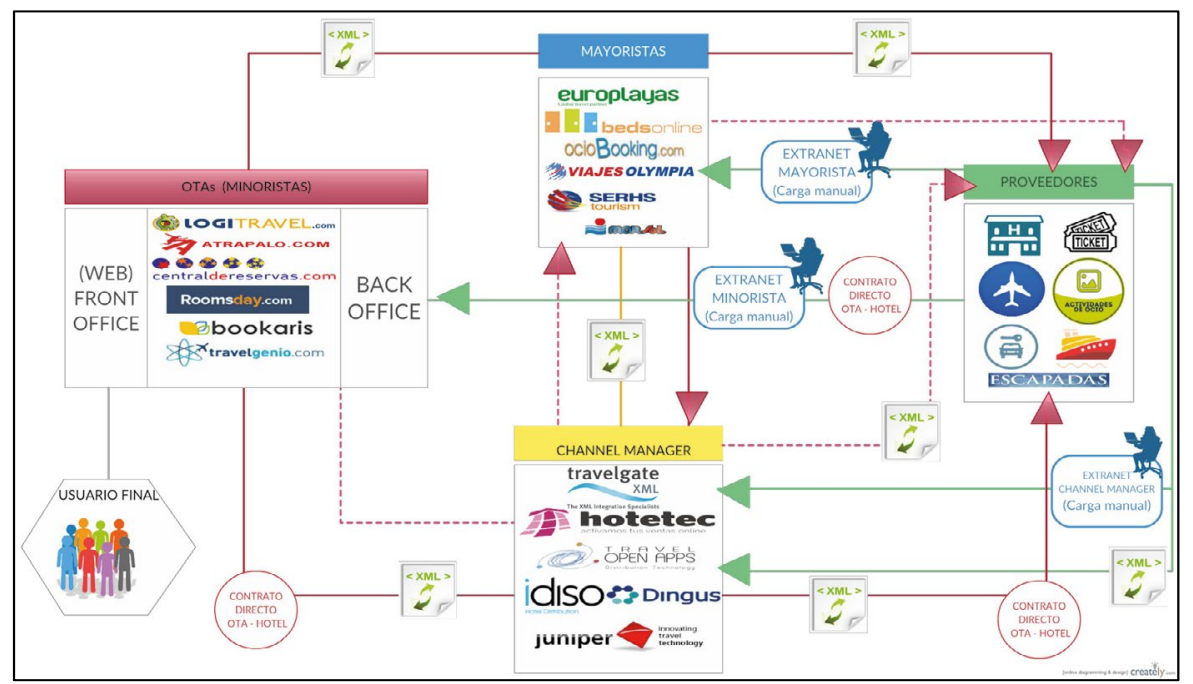

Figura 2: Proceso de adquisición de producto y/o servicio turístico. Elaboración propia.

\section{Las OTAs y los DTI}

Una vez conocidas las particularidades organizativas y de funcionamiento de las agencias de viajes online y clarificados los procesos que se establecen entre los actores implicados cabe preguntarse por la relación que deberían mantener las OTAS en el entorno de un DTI. Como se ha podido constatar, estas empresas cuentan con grandes estructuras tecnológicas que permiten la venta del destino, tanto al cliente final como a otras empresas intermediarias. Por lo tanto constituyen un canal de comercialización crucial para la venta del destino y esto no debería ser desaprovechado.

Atendiendo al autodiagnóstico propuesto por la Generalitat Valenciana (GVA, 2016) para la revisión del modelo de DTI, las OTAs se situarían en el ámbito dedicado a la Innovación y más concretamente estarían recogidas por el indicador «Penetración de TICs en empresas turísticas». Este indicador dedica una ponderación del $15 \%$ a aquellas empresas turísticas que utilicen las TICs en actividades de marketing online, social media y comercialización.

La actividad que realizan las OTAs implica al destino en tanto que es un intermediario tecnológico dedicado a la venta de productos y servicios turísticos (generalmente el alojamiento es el principal producto). No obstante, 
Actas del Seminario Internacional Destinos Turísticos Inteligentes: nuevos horizontes en la investigación y gestión del turismo

Universidad de Alicante, 26 y 27 de octubre de 2017

como se ha visto anteriormente, las OTAs realizan una importante función de asesoría y de gestión de la información y de marketing para los destinos en su tarea de negociar con proveedores y dedicarse al cliente. Por ello, entendemos que el modelo de DTI debería asimismo incluir un indicador destinado a conocer si el destino tiene voluntad por integrar a los prestatarios de servicios y más concretamente a las OTAs. Esta integración en el sistema turístico local se podría ver reflejado en los canales de comercialización y en particular a través de la web turística de los destinos permitiendo un enlace a estos prestatarios. La preocupación del destino turístico inteligente debería ser la de encontrar puntos de integración tecnológica para poder estar presente en la cartera de productos que comercializa la OTA.

Sin embargo, a pesar de la importante actividad que generan las OTAs para los destinos, la característica de ser "multiproducto» y «multidestino» hace que no sean consideradas como dinamizadoras del mismo. Se observa cómo estas empresas inician las relaciones en el destino en el que se ubican pero progresivamente, conforme van aumentando su volumen de negocio, incorporan nuevos destinos de forma que la dedicación a la comercialización "prioritaria» o "exclusiva» del destino inicial se abandona para introducir otros nuevos.

En este sentido, la aportación que hace la OTA al destino se centra en la contratación del producto el cual se realiza bien directamente con el proveedor del servicio (hoteles, empresas de alquiler de coches, compañías aéreas....) o a través de intermediarios (turoperadores, consolidadores aéreos, receptivos...).

Si se comparan las OTAs con las agencias de viajes tradicionales, se observa que éstas mayoritariamente siguen realizando una función emisora, esto es, su actividad principal consiste en la venta de paquetes combinados y otros productos para los residentes hacia otros destinos distintos del lugar donde se encuentra, con lo cual no se establece una relación directa con los destinos donde se ubican. Solo en el caso de las agencias que realmente realizan funciones receptivas complementan la oferta de otros en la venta de servicios complementarios como pueda ser conexiones a otros destinos, venta de entradas a atracciones turísticas o espectáculos, tours por la ciudad, etc. Las OTAs analizadas no demuestran una clara vocación por un destino en concreto ya que como se ha comentado están centradas en conseguir tarifas y condiciones en la negociación de grandes volúmenes de contratación. 
Actas del Seminario Internacional Destinos Turísticos Inteligentes:

nuevos horizontes en la investigación y gestión del turismo

Universidad de Alicante, 26 y 27 de octubre de 2017

No obstante, es interesante para el DTI aprovechar el potencial comercializador de las agencias de receptivo y que apuesten por la creación de paquetes turísticos dirigidos a extender el producto ofrecido y adaptarlos a las necesidades del mercado para que puedan ser comercializados, tanto a través de las OTAs como en la agencia de viajes tradicional.

En todo caso, conseguir la integración de estos actores en la gestión de un destino es fundamental en términos de compartir información. Cuando el destino negocia ha de hacerlo globalmente considerando a todos los prestatarios turísticos. El papel de los entes de gestión turística local de un DTI es el de promover la integración de la información de forma que tenga visibilidad y que puedan dar respuesta a las peticiones de los clientes recopilando la oferta de los proveedores a través de la tecnología para ponerla a disposición de los potenciales clientes. La propuesta de desarrollo pasa por aumentar las relaciones con el destino y particularmente aprovechar las OTAs que trabajan con el mismo para facilitar al cliente final la adquisición de productos y servicios turísticos.

\section{Discusión y conclusiones}

La discusión se ha planteado en torno a dos aspectos fundamentales para el funcionamiento de las OTA's, en general, y en su papel como dinamizador de un DTI. Así en primer lugar, en relación a la operativa e integración de estas empresas se concluye que no respetar la paridad de precios trae consigo importantes consecuencias las cuales pueden llegar a provocar rupturas en las negociaciones de contratación entre la OTA y el proveedor. Por otro lado, se destaca a lo largo de esta investigación la importancia de la tecnología para la realización de integraciones XML y el avance que han supuesto en la distribución turística.

En relación a estos procesos de integración tecnológicos, se destaca la aportación que han supuesto los Channel Manager como herramienta fundamental para la integración y la distribución turística. Travel Open Apps ${ }^{6}$ en la Comunidad Valenciana ha servido para simplificar el proceso de adquisición del producto, especialmente para las pequeñas empresas que no tienen

6. El impacto de Travel Open Apps en la distribución turística de la oferta turística en la Comunitat Valenciana es significativo y se observa el aumento en la adhesión de estas empresas. Los datos actualizados se pueden consultar en https://www.travelopenapps. org/travelopenapps-en-cifras/ 
Actas del Seminario Internacional Destinos Turísticos Inteligentes:

nuevos horizontes en la investigación y gestión del turismo

Universidad de Alicante, 26 y 27 de octubre de 2017

suficientes recursos tecnológicos, para que el hotel, tipo de habitación y régimen mostrado por la OTA, coincida con el ofrecido por el proveedor, ya que un error en un código supondría que el producto seleccionado por el cliente y confirmado por la OTA no coincidiría con el reservado por la OTA a su proveedor.

En relación al papel dinamizador de las OTAs en un DTI, cabe preguntarse si queremos que las agencia de viajes sean asimiladas como un elemento más de un DTI. Si es así, las OTAs deben estar integradas convenientemente entre las estrategias de marketing y comunicación del destino. Una forma de propiciar esta comunicación sería darles visibilidad a través del sitio web y aprovechar su capacidad comercializadora incluso como fuente de información actualizada otorgando mayor eficiencia al destino.

De esto se desprende un aspecto fundamental como es la necesidad de personal formado tanto en técnicas de negociación como en aspectos tecnológicos para poder afrontan tareas complejas para la realización de mapeos, mantenimiento del producto y tareas de back office relacionadas con el proceso de reservas y adquisición de productos.

\section{Referencias}

Agencia Valenciana del Turismo (2017): Destinos Turísticos Inteligentes: manual Operativo para la configuración de Destinos Turísticos Inteligentes. Recuperado el 20 de mayo de 2017. http://invattur.gva.es/proyecto-ic/destinos-turisticos-inteligentes-comunitat-valenciana/

Del Alcázar Martinez, B. (2002). Los canales de distribución en el sector turístico. ESIC. Madrid.

Buying, M. (2015). Emarketer.com. Recuperado el 16 de Agosto de 2016, de emarketer.com: <http://www.emarketer.com/Article/Social-Network-Ad-RevenuesAccelerate-Worldwide/1013015>

Comisión Europea (2012). Tourismlink. Linking tourism professionals with the digital market. Recuperado el 20 de marzo de 2017. http://www.hotrec.eu/Documents/Document/20120521114433-TOURISMlink_brochure_final.pdf

Comisión Nacional de los Mercados y la Competencia (2014): Informe sobre el comercio electrónico en España a través de entidades de medios de pago. Primer trimestre 2014. Recuperado el 30 de marzo de 2017. https://www.cnmc. es/sites/default/files/1529913_5.pdf 
Actas del Seminario Internacional Destinos Turísticos Inteligentes:

nuevos horizontes en la investigación y gestión del turismo

Universidad de Alicante, 26 y 27 de octubre de 2017

Huerta, J. M. (2016). Gestionati.es. Recuperado el 19 de Junio de 2016, de gestionati. es: <http://gestionati.es/comentarios/el-estandar-ota-una-historia-sobre- protocolos-e-integraciones>

Laborda Vallespín, A. (2015). mglobalmarketing.es. Recuperado el 22 de Julio de 2016, de mglobalmarketing.es: <http://mglobalmarketing.es/blog/como-se-define-el-branding-de- una-marca/>

Maciá Domene, F., \& Gosende Grela, J. (2012). Posicionamiento en buscadores. Anaya multimedia. Madrid.

Nielsen, J. (2011). nngroup.com. Recuperado el 11 de Agosto de 2016, de nngroup. com: <https://www.nngroup.com/articles/how-long-do-users-stay-on-webpages/>

Ormos. (11 de Enero de 2016). Las disparidades de precios en OTA y metabuscadores, en aumento. Recuperado el 21 de Julio de 2016, de Hosteltur.com: <http://www.hosteltur.com/114491_disparidades-precios-ota-metabuscadores- aumento.html>

Segittur. (2016). Segittur.es. Recuperado el 18 de Julio de 2016, de <http://www. segittur.es/opencms/export/sites/segitur/.content/galerias/descargas/proyecto s/Glosario_tecnologico_turistico.pdf> 